\title{
Implementación de la publicación anticipada en la Revista Médica Panacea
}

\author{
IMPLEMENTATION OF THE EARLY PUBLICATION IN THE REVISTA MÉDICA PANACEA
}

\author{
J. Jhonnel Alarco ${ }^{1, a}$, Jorge Ybaseta-Medina,b \\ Revista Médica Panacea ${ }^{1}$ \\ Editor asociadoa, Jefe de la revistab
}

a rapidez del intercambio de información es fundamental en todos los campos científicos y si esta
información se puede compartir libremente sin costo alguno para los autores y lectores, tenemos la
fórmula perfecta para difundir información científica (1).

La publicación anticipada (Ahead of Print), se define como aquella que ocurre antes de que se publique la versión impresa, lo que significa que solamente se puede conseguir si la revista dispone de una versión electrónica ya que en su versión en papel puede tardar varios meses en aparecer publicada. El termino EPub (electronically published) Ahead of Print, significa publicado electrónicamente antes de su impresión y es una forma de poder comunicar las investigaciones en un menor tiempo.

Packer (2) refiere que la publicación anticipada agrega valor a dos funciones clave de este proceso, atiende la demanda básica de que los autores vean su investigación evaluada por pares y comunicada lo más rápido posible y actualiza más velozmente el espacio del conocimiento científico. Las ventajas de la publicación anticipada se detallan en la Tabla 1.

\section{TABLA 1 Ventajas de la publicación anticipada}

- Permite a los lectores acceder a los documentos más recientes del campo de su investigación.

- Los plazos entre la presentación y publicación de los artículos se reducen considerablemente, lo que conviene al autor.

- Los artículos publicados en forma anticipada (ahead of print) pueden ser indizados, citados, etc.

- Cuanto más rápido los artículos son considerados por los índices internacionales, más rápido están visibles aumentando el tiempo de exposición y la posibilidad de citación.

- Aliada al acceso abierto, la publicación anticipada acelera la disponibilidad universal de la investigación científica.

Actualmente esta forma de publicación es adoptada por varias casas editoriales y revistas de prestigio $(3,4)$ y es parte de lo que se conoce como publicación continua, es decir que los artículos se publican a medida que el proceso editorial y de publicación concluyen.

La Revista Médica Panacea (RMP) ha adoptado esta novedosa forma de publicación, lo que significa que en adelante los artículos se publicaran mucho antes de los plazos comunes, es decir que no se tiene que esperar hasta la fecha de la publicación de la versión impresa, se publicarán, conforme sean aceptados en su versión final.

Se ha decidido que las versiones anticipadas tengan el mismo formato que la versión final (de impresión), solamente con algunas variaciones sobre la paginación, sobre las fechas de publicación y con un pie de pagina que indique que se trate de una publicación anticipada.

De esta manera se garantiza que los tiempos de espera se reducirán considerablemente, lo que seria mas atractivo para autores que necesiten que sus artículos se publiquen mas rápidamente, lo que actualmente resulta ser casi una constante en la investigación científica. 
Además, según los requisitos de uniformidad para manuscritos enviados a revistas biomédicas, dictadas por el Comité Internacional de Editores de Revistas Médicas (ICMJE) (5) conocido como las Normas de Vancouver (utilizado por la revista), existe un estilo definido para citar artículos publicados en formato electrónico antes que en su versión impresa:

Sait KH, Ashour A, Rajabi M. Pregnancy outcome in non-gynecologic cancer. Arch Gynecol Obstet. 2004 Jun 2 [Epub ahead of print].

Y una vez salga la versión impresa esta citación queda modificada como sigue:

Sait KH, Ashour A, Rajabi M. Pregnancy outcome in non-gynecologic cancer. Arch Gynecol Obstet. 2005 Apr; 271(4): 346-9. Epub 2004 Jun 2.

En la versión en español adoptada por la RMP, su equivalente seria:

Gutierrez-Munares ME, Martinez-Pariona PE, Apaza-Rodrigo JH. Frecuencia de los factores de riesgo obstétricos en la ruptura prematura de membranas pretermino, Hospital Regional de Ayacucho, Perú. Rev méd panacea.2014; 20 de Noviembre de 2014 [publicado electrónicamente antes de su impresión]

Y la citación para la versión impresa seria de la siguiente forma:

Gutierrez-Munares ME, Martinez-Pariona PE, Apaza-Rodrigo JH. Frecuencia de los factores de riesgo obstétricos en la ruptura prematura de membranas pretermino, Hospital Regional de Ayacucho, Perú. Rev méd panacea.2014; 4(3):70-73. Publicado electrónicamente 20 de Noviembre de 2014 .

En la figura 1, se puede observar la media de los tiempos de espera en los últimos cuatro años de funcionamiento de la revista, desde el envío del manuscrito hasta su aceptación (E-A), desde su aceptación hasta su publicación (A-P) y el tiempo promedio desde el envío hasta su publicación final (EP). Hemos notado que el máximo tiempo de espera fue de 9,8 meses y el mínimo fue de 0,5 meses, con un promedio de 3,8 meses, lo que es un tiempo de espera que podría considerarse moderado, incluso menor que otras revistas.

Con la nueva estrategia de publicación anticipada, en cuanto el manuscrito sea aceptado para su publicación, creemos que los tiempos de demora se reducirán a la mitad, lo que significa que la publicación ocurrirá en un lapso de dos meses aproximadamente.

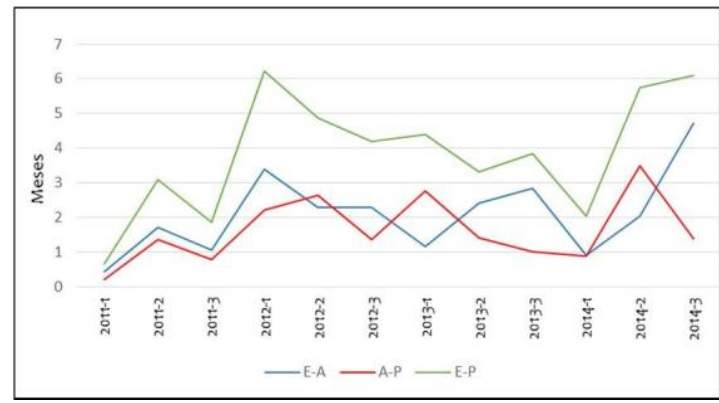

FIGURA 1. Tiempos promedios de publicación desde el envio del manuscrito hasta la aceptacion (E-A), desde la aceptación hasta la publicación (A-P) y desde el envio hasta la publicación (E-P) en los cuatro años de funcionamiento de la Revista Médica Panacea.

\section{REFERENCIAS BIBLIOGRÁFICAS}

1. González-Méijome JM. Journal of Optometry goes EPub Ahead of Print!. Journal of Optometry. 2012;5(1):1.

2. Packer Abel L. El modus operandi online y el avance de los periódicos brasileños de enfermería. Acta paul. enferm. 2012; $25(2)$.

3. Patel P. More research, faster publication - Papers in Press and Continuous Publishing. En: A Blog from the publishing team at the Society of General Microbiology. London: Society of General Microbiology 2013.

4. Bachelet VC, Cardemil F. Medwave adopts continuous publication. Medwave 2013;13(3).

5. Requisitos de Uniformidad para manuscritos enviados a revistas biomédicas: Redacción y preparación de la edición de una publicación biomédica. Revista Cubana de Salud Pública. 2012;38(2):300-343.
CITA SUGERIDA.

Alarco JJ, Ybaseta-Medina J, Implementación de la publicación anticipada en la Revista Médica Panacea [Editorial]. Rev méd panacea. 2015; 5 (1):1-2. 